\title{
Suppression of LO phonon scattering in Landau quantized quantum dots
}

\author{
B. N. Murdin, A. R. Hollingworth, M. Kamal-Saadi, and R. T. Kotitschke \\ Department of Physics, University of Surrey, Guildford, Surrey GU2 5XH, United Kingdom \\ C. M. Ciesla, C. R. Pidgeon, and P. C. Findlay \\ Department of Physics, Heriot Watt University, Edinburgh, EH14 4AS, United Kingdom \\ H. P. M. Pellemans and C. J. G. M. Langerak \\ FOM-Institute for Plasma Physics “Rijnhuizen,'” P.O. Box 1207, NL-3430 BE Nieuwegein, The Netherlands \\ A. C. Rowe and R. A. Stradling \\ Solid State Group, Department of Physics, Imperial College, London, SW7 2AZ, United Kingdom \\ E. Gornik \\ Institut für Festkörperelektronik, Technische Universität Wien, A-1040 Wien, Austria
}

(Received 6 January 1999)

\begin{abstract}
Picosecond time-resolved far-infrared measurements are presented of the scattering between conductionband states in a doped quasi quantum dot. These states are created by the application of a magnetic field along the growth direction of an InAs/AlSb quantum well. A clear suppression of the cooling rate is seen, from $10^{12} \mathrm{~s}^{-1}$ when the level spacing is equal to the phonon energy, to $10^{10} \mathrm{~s}^{-1}$ away from this resonance, and thus the results provide unambiguous evidence for the phonon bottleneck. Furthermore, the lifetimes had only weak dependence on temperature between 4 and $80 \mathrm{~K}$. [S0163-1829(99)50612-7]
\end{abstract}

Electronic lifetimes of two-dimensional (2D) systems in magnetic fields are of fundamental interest in part because the quantization effect of the magnetic field mimics the effect of a quantum dot potential with an easily variable degree of confinement. ${ }^{1}$ The magnetic field perpendicular to the layers forces the electrons into confined orbits and the density of states becomes a ladder of broadened $\delta$ functions similar to that of a quantum dot. Recently much work has been carried out on the so-called "phonon bottleneck" that has been claimed to inhibit the cooling of carriers in quantum dots when the level separation is not equal to the phonon energy. ${ }^{2-6}$ However, partly as a result of different groups using different growth techniques for interband photoluminescence samples and partly on fundamental grounds, this is controversial and is the subject of much debate. ${ }^{7-12}$ Indeed several mechanisms have been proposed that may bypass the bottleneck, such as multiphonon scattering, ${ }^{7}$ Auger processes, ${ }^{8}$ excitonic effects, ${ }^{9}$ and defect related processes. ${ }^{10}$ In the present work we observe clear phonon suppression in $n$-type quasi 0D dots (i.e., Landau quantized rather than spatially quantized) by a time-resolved intraband absorption measurement. This provides unambiguous evidence for the phonon bottleneck effect independently of arguments concerning which processes dominate in the interband photoluminescence measurements in $\operatorname{dots}^{3-6,9-12}$ and quasi dots ${ }^{13}$ such as electron-hole scattering. Further, because of the very clean model system (much sharper interfaces and no wetting layer, etc.), the interpretation is not complicated by detailed questions about different growth techniques and the quality of different dot sample structures. The results should assist in the understanding of which aspect of these processes is fundamental and which is dependent on sample structure.
Resonant absorption of longitudinal optic (LO) phonons causes a variety of transport properties to oscillate with applied magnetic field, such as the magnetoresistance. ${ }^{14}$ Resonant phonon scattering occurs when

$$
\Delta l \hbar \omega_{c}=\hbar \omega_{\mathrm{LO}}
$$

where $\hbar \omega_{\mathrm{LO}}$ is the LO phonon energy, $\hbar \omega_{c}=\hbar e B / m^{*}$ is the cyclotron energy, and $\Delta l$ is an integer. At these resonances the LO phonon absorption/emission probability is strongly enhanced giving rise to large changes in the electron energy relaxation lifetime. ${ }^{15,16}$ In the present work we have used the pump-probe technique, with cyclotron resonance, to quantitatively determine the relaxation time associated with the phonon bottleneck effect away from resonance. We show additionally that the phonon bottleneck is temperature independent at least up to $80 \mathrm{~K}$.

Bleaching of the absorption of a harmonic ladder of states is impossible since the populations of adjacent states can never be equal for the entire ladder, i.e., further absorption is always possible. Nonlinear optical measurements of the lifetime, i.e., saturation or pump-probe techniques, are therefore only possible when some form of anharmonicity terminates photon absorption at some point. Because of the lack of band nonparabolicity, previous saturation measurements on GaAs quantum wells were limited to a small range of energies determined by the requirement that either the resonant polaron effect ${ }^{17,18}$ or an anticrossing with other subbands ${ }^{19}$ terminates the Landau level ladder. In the former case, for example, the measurement is only possible if the state originally at $\hbar \omega_{\mathrm{LO}}$ is bent strongly off resonance and does not therefore take part in the relaxation process. This requires 


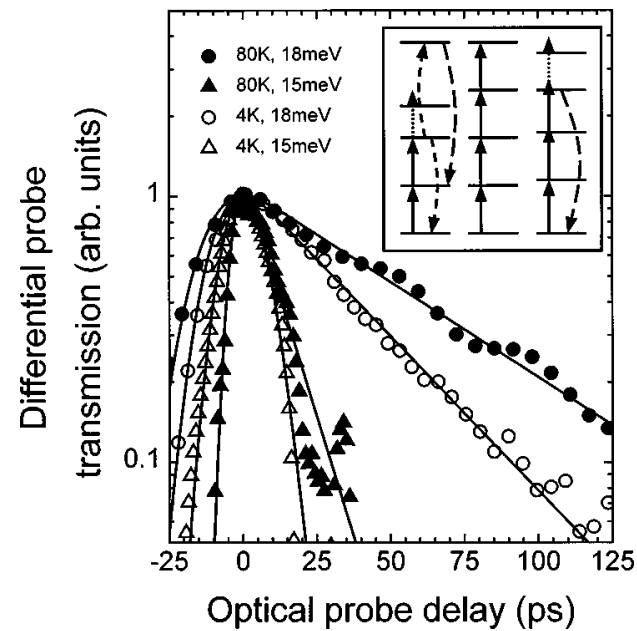

FIG. 1. Differential probe transmission vs optical delay at $T$ $=4 \mathrm{~K}$ (open symbols) and $80 \mathrm{~K}$ (filled symbols). Results were taken at $\hbar \omega_{0}=15 \mathrm{meV}$ (triangles), i.e., close to the $\Delta l=2$ resonant phonon scattering condition (which occurs at $14.8 \mathrm{meV}$ ), and at $\hbar \omega_{c}=18 \mathrm{meV}$ (circles), i.e., away from the resonance. The solid lines are results from the rate equation model as described in the text. The inset shows the following: center: a harmonic ladder (no saturation); left: polaron effect (at $\Delta l=3$ ) makes the fourth level nonresonant, but Auger processes (short dash) followed by LO phonon scattering (long dash) cause relaxation; right: band nonparabolicity terminates excitation (number of levels included depends on the linewidth).

samples having very narrow linewidths with low enough carrier concentration not to screen the polaron effect. The harmonicity of the rest of the ladder means that Auger (electronelectron) processes will eventually raise carriers above the phonon energy after which a fast deexcitation can occur (this is shown schematically in the inset of Fig. 1). The electronelectron part of the sequential process has been shown to be the slowest (i.e., dominating) leg from the observation of an $n$ dependence of the lifetime. ${ }^{17,20}$

We show here a combination of pump-probe and saturation measurements of the cyclotron resonance in an InAs/ $\mathrm{AlSb}$ quantum well. This narrow gap material $\left(E_{g}\right.$ $=0.42 \mathrm{eV}$ in InAs) has sufficient band nonparabolicity so that higher Landau levels are bent out of resonance (see Fig. 1 inset). The Landau level ladder is thus truncated and saturation is possible over a wide range of energies. This enables determination of the energy relaxation lifetime both below and above the LO phonon energy, even with carrier concentrations high enough to suppress the polaron effect.

The sample studied was a 15-nm InAs quantum well, grown between AlSb barriers by molecular beam epitaxy on an undoped GaAs substrate. Thick strain relaxing layers of $0.8-\mu \mathrm{m} \mathrm{GaSb}$ and $0.8-\mu \mathrm{m} \mathrm{AlSb}$ followed by a 10 period superlattice of (2.5-nm AlSb, 2.5-nm GaSb) were grown on the substrate. The 20-nm AlSb barrier, 15-nm InAs well, and 15-nm AlSb barrier were capped with 12-nm GaSb. The well/barrier interfaces were InSb-like. The carrier density at helium temperatures has been determined by transport measurements to be $5 \times 10^{11} \mathrm{~cm}^{-2}\left(8.5 \times 10^{11} \mathrm{~cm}^{-2}\right.$ at $\left.77 \mathrm{~K}\right)$ with a mobility of $262000 \mathrm{~cm}^{2} / \mathrm{V} \mathrm{s}$. The electron effective mass, as determined from the cyclotron absorption, was $0.035 m_{0}$. The picosecond resolution measurements were performed

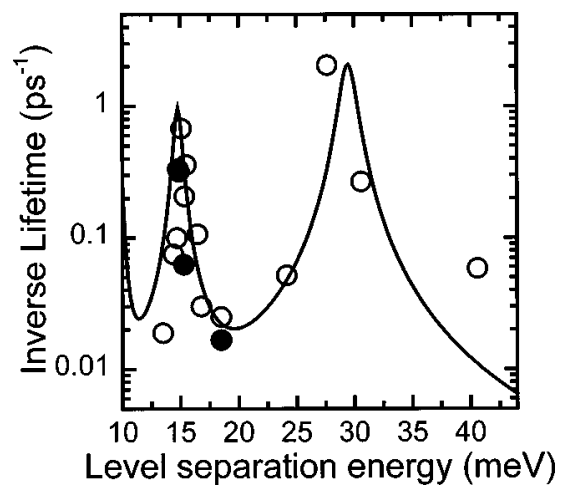

FIG. 2. Inverse decay time vs level separation energy. Experimentally determined data at $T=4 \mathrm{~K}$ (open circles) and $80 \mathrm{~K}$ (filled circles). The calculated scattering rate is shown as the solid line.

using the far-infrared, Free Electron Laser for Infrared eXperiments (FELIX) in The Netherlands. ${ }^{21}$ The laser is continuously tunable in stages from 5-100 $\mu \mathrm{m}$ and delivers "macropulses" of typically $4-\mu$ s duration at a $5-\mathrm{Hz}$ frequency. Each macropulse consists of a train of micropulses separated by $40 \mathrm{~ns}$, which have an energy of $1-10 \mu \mathrm{J}$. The micropulse length is controllable between $1-10 \mathrm{ps}$.

The sample was mounted in vacuum on a cold finger, in the Faraday configuration of a horizontal bore superconducting magnet. In the saturation experiments the laser frequency was fixed and the transmission was measured versus field for different laser intensities. The cyclotron absorption was bleached at high intensity and the lowest laser frequency for which this was fully achieved was $13.7 \mathrm{meV}$. The highfrequency limit was determined by the magnet and was 40 $\mathrm{meV}$. For the pump-probe experiments the laser was split into two beams, and calibrated wire-mesh attenuators were used to independently control the intensities so as to achieve full saturation by the pump but linear absorption only by the probe. The intensities used were estimated to be typically 100 and $1 \mathrm{~kW} \mathrm{~cm}^{-2}$ for the pump and probe, respectively. For a given fixed magnetic field, the pump-probe experiments were performed with the laser fixed at the cyclotron frequency. Using $\hbar \omega_{\mathrm{LO}}=29.5 \mathrm{meV}$ for InAs, the resonant phonon scattering [Eq. (1)] occurs at $\hbar \omega_{c}=29.5,14.8,9.8$ meV etc. Example pump-probe results are shown in Fig. 1 for sample temperatures of 4 and $80 \mathrm{~K}$ both near to resonance at $15 \mathrm{meV}$ and away from it, at $18 \mathrm{meV}$. When the laser was detuned from the cyclotron frequency the pumpprobe bleaching effect disappeared. The inverse lifetimes derived from the decay curves are shown as a function of level separation in Fig. 2. Both the results for $4 \mathrm{~K}$ and $80 \mathrm{~K}$ are shown. The scattering rate away from resonance is strongly suppressed compared with that on resonance. Although there is a clear difference in the lifetimes at 4 and $80 \mathrm{~K}$, there is little difference in the suppression factor. The paradoxical result that the lifetime increases with temperature at low temperatures has been predicted previously for 0D states ${ }^{16}$ and is actually observed here. We discuss the temperature dependence below.

We have calculated the inter-Landau level scattering probability by LO phonon emission alone ${ }^{16,22}$ using the wave functions from a 14-band $\mathbf{k} \cdot \mathbf{p}$ calculation, shown in Fig. 3. The full width at half maximum broadening of the matrix element of the LO interaction was taken to be $0.3 \mathrm{meV}$, a 


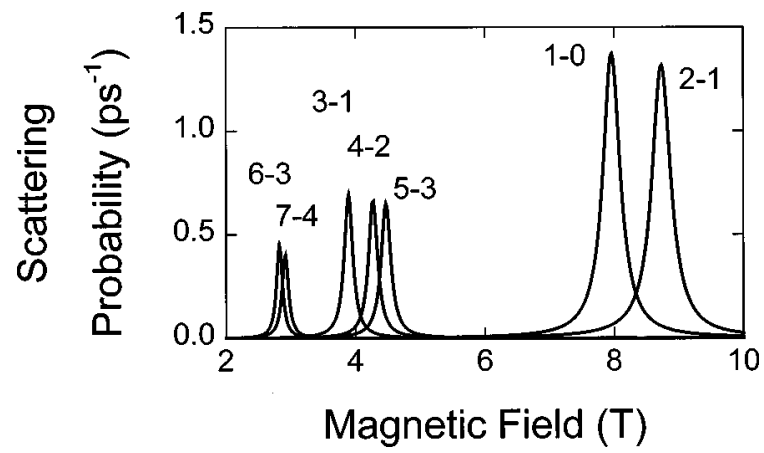

FIG. 3. Electron-LO phonon scattering probability vs field calculated for an InAs/AlSb quantum well with a mobility $\mu$ $=262000 \mathrm{~cm}^{2} / \mathrm{V} \mathrm{s}$. The spin splitting has not been resolved here. The rates shown are for empty Landau levels, i.e., occupation effects, etc. are included later in the calculation via Eq. (2). Only selected transitions are shown, those near to the fill factors relevant for our sample.

value one fifth of that used for a sample with about five times lower mobility. ${ }^{16}$ The LO phonon scattering rate depends strongly upon the level separation and has resonances according to Eq. (1). The scattering rate also varies from resonance to resonance and decreases with increasing $\Delta l$. Thus the $\Delta l=1$ resonance at $B \sim 8 \mathrm{~T}$ is stronger than for $\Delta l=2$ at $B \sim 4 \mathrm{~T}$. We emphasize that the variation from resonance to resonance in the quasi dot calculation parallels the real dot situation where the phonon relaxation may be significantly suppressed by a reduction in the matrix element between the phonon and electronic states (this is in addition to the obvious suppression required by energy conservation). Transitions between successively higher pairs of levels are pushed to higher fields by the nonparabolicity. These scattering rates are fed into a rate equation model for the level filling factors, which has no free parameters, as follows:

$$
\frac{d f_{l}}{d t}=\Phi\left[A_{l-1}-A_{l}\right]-\sum_{l^{\prime}<l}\left[\frac{f_{l}\left(1-f_{l^{\prime}}\right)}{\tau_{l l^{\prime}}}\right]+\sum_{l^{\prime}>l}\left[\frac{f_{l^{\prime}}\left(1-f_{l}\right)}{\tau_{l^{\prime} l}}\right],
$$

where $\Phi$ is the photon flux density, $A_{l}$ is the absorption from level $l \rightarrow l+1$, and $\tau_{l l^{\prime}}$ are the LO phonon lifetimes from Fig. 3. $A_{l}$ can be found from consideration of a conducting sheet with the Drude conductivity $\sigma_{l}$ (which contains the Lorentzian dependence on magnetic field) on top of a dielectric substrate. ${ }^{17}$ The total transmission, $T_{\text {tot }}\left(\Sigma \sigma_{l}\right)$ as a function of time is shown as the solid lines in Fig. 1, and the inverse decay time of $T_{\text {tot }}$ is shown as the solid line in Fig. 2. Excellent agreement is found at all except the highest energy, where high reststrahlen reflections made measurements more difficult. The model involves only one fitting parameter, the broadening, and the populations do depend upon the measured absorption cross section and intensity. However, the predicted pump-probe decay times are relatively insensitive to the latter two parameters as long as full saturation is achieved. The scattering off-resonance can have contributions from mechanisms other than LO phonon scattering such as acoustic phonon emission, particularly for narrower linewidths, and this requires further investigation.

Our results and calculation for InAs/AlSb show lifetimes at resonance with Eq. (1) that are consistent with those found previously in InAs/GaSb, ${ }^{15,16}$ where lifetimes on resonance were subpicosecond. However, in these earlier experiments the pump-probe technique was not available (giving considerable systematic uncertainty), and the sample used had a much lower mobility $\left(40000 \mathrm{~cm}^{2} / \mathrm{V} \mathrm{s}\right)$ and broader linewidth as compared with the present work. Under these circumstances it was not possible to observe the strong phonon bottleneck effect or accurately measure the lifetime away from the phonon resonances as reported here. Furthermore, measurements at the higher temperatures were not attempted.

The values for the lifetimes away from the phonon resonances are consistent with previous measurements of interLandau level relaxation in GaAs heterostructures. As mentioned above, a scattering rate proportional to carrier density (i.e., $\tau \propto 1 / n$ ) was found, and therefore associated with electron-electron scattering. ${ }^{17}$ The very long lifetimes of around $1 \mathrm{~ns}$, when extrapolated to our higher density would lead to much shorter times. However, our data agree very well with a model with only one process that affects the energy of the electron system, the LO phonon emission (and absorption). We implicitly assume a certain degree of energy redistribution processes such as electron-electron and ionized impurity scattering, which broaden the levels. The relaxation proceeds via the latter two to the phonon energy, followed by phonon emission. Off resonance, the number of states in the wings of the levels overlapping with the phonon energy is small giving a lower net LO phonon rate. This would be particularly true in higher mobility samples, in which case it may be possible to observe other processes, such as acoustic phonon scattering, and at energies larger than $\hbar \omega_{\text {LO }}$ multiphonon events would be possible.

By analogy with the present OD case, it is known that intersubband relaxation in quantum wells is strongly dependent on the subband separation, and theoretical models of 2D intersubband relaxation have shown enhanced cooling where the quantum well subband separation energy equals the LO phonon energy. ${ }^{23}$ The existence of a bottleneck in the cooling of carriers between subbands in wide quantum wells when the subband separation is smaller than the LO phonon energy has been observed previously. ${ }^{24}$ Lifetimes of the order of 50 ps due to acoustic phonon emission were found at low temperatures. In this system (with zero magnetic field) there are always states that exist at the phonon energy but away from the subband minimum, and thus the bottleneck may be overcome by increasing the temperature above some activation threshold, $35 \mathrm{~K}$ in that case. In the present $0 \mathrm{D}$ system, raising the temperature does not remove the bottleneck effect even up to $80 \mathrm{~K}$.

Our model also explains the temperature dependence of our results. Saturation occurs at a fixed electron distribution that depends only on the level structure and optical matrix elements, i.e., is independent of the relaxation process or of lattice temperature. This gives a paradoxical result that when the temperature is increased, the cooling lifetime increases, since our initial (saturated) excited electron distribution is then less different from equilibrium. ${ }^{16}$ At the highest temperatures investigated here, $k_{B} T \sim \hbar \omega_{\mathrm{LO}} / 5$ and the population of LO phonons is starting to become important, and we have indeed observed a slight rise in lifetime. However, as the temperature is increased further, increased broadening would enhance the scattering off-resonance thus reversing this 
trend. In our case no changes in the cyclotron linewidth or the mobility up to $80 \mathrm{~K}$ occur, but above this point the mobility starts to drop $(\mu=20000 \mathrm{~cm} / \mathrm{V} \mathrm{s}$ at $300 \mathrm{~K})$. Some interband photoluminescence (PL) experiments have also shown lifetimes decreasing with temperature, ${ }^{5}$ possibly due to additional processes not present in our quasi dots such as Auger involving holes or barrier electrons.

In summary, we have measured the relaxation lifetime of carriers in a quasi quantum dot resulting from a twodimensional electron gas in a magnetic field. The measurements have been made both such that the level separation equals the phonon energy and away from this condition. A bottleneck exists in LO-phonon cooling of carriers away from the resonance, which is independent of temperature between 4 and $80 \mathrm{~K}$.

The fact that the LO phonon cooling is significantly quenched away from the resonant phonon scattering condition, may have important consequences for quantum dot devices based on either inter- or intraband optical transitions, since it confirms the existence of the LO-phonon bottleneck between the lowest dot states, even at the higher temperatures. For comparison with spatial confinement, the typical dot size with level separation corresponding to the fundamental resonance at $29.5 \mathrm{meV}$ is of the order of $50 \mathrm{~nm}$. On the other hand, the lifetimes found here under bottleneck conditions ( $\sim 50 \mathrm{ps})$ may not be deleterious since they are similar to the lifetimes predicted to be necessary for dot laser operation. ${ }^{11}$ In any case, several mechanisms have been proposed that efficiently bypass the phonon bottleneck in the electron cooling in real dots. In particular, under high energy interband excitation electron-hole scattering may dominate, but also scattering with continuum electrons, cascading through nonradiating excitonic states, tunneling between adjacent dots and confined phonons have been suggested (see, for example Refs. 7-10). The present determination of the scattering rate between the lowest states in a doped quasi quantum dot structure (without the complication of holes) should help in the quantitative evaluation of these fundamental processes.

The authors wish to thank Dr. A. van der Meer and the FELIX staff for skillful technical assistance. We are grateful to Ivair Gontijo and Ray Murray for helpful discussions concerning the "phonon bottleneck" problem. This work was undertaken as part of the joint EPSRC (UK) and FOM (NL) program at FELIX. C.J.G.M.L. and H.P.M.P. are grateful for the support of the EPSRC (UK). Three of us (A.R.H., C.M.C., and P.C.F.) are grateful to the EPSRC for support and for CASE awards with DERA, Malvern. A.C.R. is grateful to the Commonwealth for support.
${ }^{1}$ U. Bockelmann, Phys. Rev. B 50, 17271 (1994).

${ }^{2}$ U. Bockelmann and G. Bastard, Phys. Rev. B 42, 8947 (1990).

${ }^{3}$ H. Benisty, C. M. Sotomayor-Torres, and C. Weisbuch, Phys. Rev. B 44, 10945 (1991).

${ }^{4}$ K. Brunner et al., Phys. Rev. Lett. 69, 3216 (1992).

${ }^{5}$ K. Mukai et al., Phys. Rev. B 54, R5243 (1996).

${ }^{6}$ K. H. Schmidt et al., Phys. Rev. B 54, 11346 (1996).

${ }^{7}$ T. Inoshita and H. Sakaki, Phys. Rev. B 46, 7260 (1992).

${ }^{8}$ U. Bockelmann and T. Egeler, Phys. Rev. B 46, 15574 (1992).

${ }^{9}$ U. Bockelmann et al., Phys. Rev. Lett. 76, 3622 (1996).

${ }^{10}$ D. F. Schroeter, D. J. Griffiths, and P. C. Sercel, Phys. Rev. B 54, 1486 (1996).

${ }^{11}$ M. J. Steer et al., Phys. Rev. B 54, 17738 (1996).

${ }^{12}$ R. Heitz et al., Phys. Rev. B 57, 9050 (1998).

${ }^{13}$ C. Hartmann et al., Phys. Rev. Lett. 80, 810 (1998).
${ }^{14}$ D. J. Barnes et al., Phys. Rev. Lett. 66, 794 (1991).

${ }^{15}$ T. A. Vaughan et al., Phys. Rev. B 53, 16481 (1996); B. N. Murdin et al., Phys. Status Solidi B 204, 155 (1997).

${ }^{16}$ X. Wu and F. M. Peeters, Phys. Rev. B 55, 9333 (1997).

${ }^{17}$ I. Maran et al., Semicond. Sci. Technol. 9, 700 (1994).

${ }^{18}$ W. Heiss et al., Appl. Phys. Lett. 67, 1110 (1995).

${ }^{19}$ L. S. Muratov et al., Superlattices Microstruct. 21, 501 (1997).

${ }^{20}$ E. Gornik et al., Phys. Rev. Lett. 40, 1151 (1978).

${ }^{21}$ D. Oepts, A. F. G. van der Meer, and W. van Amersfoot, Infrared Phys. Technol. 36, 297 (1995).

${ }^{22}$ P. Warmenbol et al., Phys. Rev. B 40, 6258 (1989).

${ }^{23}$ S. C. Lee, I. Galbraith, and C. R. Pidgeon, Phys. Rev. B 52, 1874 (1995).

${ }^{24}$ B. N. Murdin et al., Phys. Rev. B 55, 5171 (1997). 